\title{
LINEAR FUNCTIONALS OF FOLIAGE ANGLE DENSITY
}

\author{
R. S. ANDERSSEN and D. R. JACKETT ${ }^{1}$
}

(Received 1 March 1982; revised 6 January 1983)

\begin{abstract}
Knowledge about the foliage angle density $g(\alpha)$ of the leaves in the canopy of trees is crucial in foresty mangement, modelling canopy reflectance, and environmental monitoring. It is usually determined from observations of the contact frequency $f(\beta)$ by solving a version of the first kind Fredholm integral equation derived by Reeve (Appendix in Warren Wilson [22]). However, for inference purposes, the practitioner uses functionals defined on $g(\alpha)$, such as the leaf area index $F$, rather than $g(\alpha)$ itself. Miller [12] has shown that $F$ can be computed directly from $f(\beta)$ without solving the integral equation. In this paper, we show that his result is a special case of a general transformation for linear functionals defined on $g(\alpha)$. The key is the existence of an alternative inversion formula for the integral equation to that derived by Miller [11].
\end{abstract}

\section{Introduction}

The foliage angle density $g(\alpha)$ (i.e. the area per unit volume of foliage inclined at an angle $\alpha$ to the horizontal) is related to the contact frequency $f(\beta)$ (i.e. the observed percentage of foliage in the canopy as a function of the elevation angle $\beta$ ) by the following first kind Fredholm integral equation:

$$
f(\beta)=\int_{0}^{\pi / 2} K(\alpha, \beta) g(\alpha) d \alpha \quad(0 \leqslant \beta \leqslant \pi / 2)
$$

with kernel

$$
K(\alpha, \beta)=\left\{\begin{array}{ll}
\cos \alpha \sin \beta & (\alpha \leqslant \beta), \\
\cos \alpha \sin \beta\{1+2(\tan \psi(\alpha, \beta)-\psi(\alpha, \beta)) / \pi\} & (\beta \leqslant \alpha),
\end{array}\right\}
$$

\footnotetext{
${ }^{1}$ Division of Mathematics and Statistics, CSIRO, P.O. Box 1965, Canberra City, A.C.T. 2601.

(c) Copyright Australian Mathematical Society 1984, Serial-fee code 0334-2700/84
} 
where

$$
\left.\psi(\alpha, \beta)=\cos ^{-1}(\tan \beta / \tan \alpha) \quad \text { (principal value; } \alpha \geqslant \beta\right) .
$$

This version of Reeve's equation (Appendix in Warren Wilson [22]) was derived by Philip [13], page 359. Miller [11] made a detailed study of this equation and among other results obtained, after imposing suitable regularity conditions on $f(\beta)$, the following inversion formula

$g(\alpha)=\tan \alpha \sec ^{3} \alpha \int_{\alpha}^{\pi / 2} \frac{3 \cos ^{2} \tau \sin \tau\left(f(\tau)+f^{\prime \prime}(\tau)\right)-\cos ^{3} \tau\left(f^{\prime}(\tau)+f^{\prime \prime \prime}(\tau)\right)}{\left(\tan ^{2} \tau-\tan ^{2} \alpha\right)^{1 / 2}} d \tau$,

where $f^{\prime}(\tau)=d f(\tau) / d \tau$.

The improperly posed nature of (1) and (2) (cf. de Hoog [6] and Smith, Oliver and Berry [17], Appendix) is a direct consequence of (4) since it involves first, second and third derivatives of the contact frequency $f(\beta)$. In particular, the form of the foliage angle density $g(\alpha)$ will be acutely sensitive to small perturbations in the contact frequency $f(\beta)$. As a direct consequence, it will be necessary to introduce some form of "stabilization" into either the direct solution of (1) and (2) or the evaluation of the inversion formula (4) in order to cope with the sensitivity which results from the use of observational data

$$
\begin{array}{r}
\left\{d_{j}\right\}=\left\{d_{j} \mid d_{j}=f\left(\beta_{j}\right)+\varepsilon_{j}, j=1,2, \ldots, n, 0<\beta_{1}<\beta_{2}<\cdots<\beta_{n}\right. \\
\left.\varepsilon_{j} \equiv \text { random error }\right\}
\end{array}
$$

to represent $f(\beta)$. In fact, it follows immediately from (4) that the sensitivity arises from the third differentiation which must be applied to the data $\left\{d_{j}\right\}$.

Thus, if order of differentiation is taken as the measure of sensitivity ( $c f$. Wahba [21]), it follows that the present problem will require stronger stabilization than that required for the solution of Abel integral equations (half differentiation) and the direct numerical differentiation (first derivative) of the data $\left\{d_{j}\right\}$.

Stabilization can be introduced in a number of ways. It can be applied directly to either the numerical solution of (1), (2) and (5) (e.g. regularization ( $c f$. Wahba [20] and Lukas [10])) or the evaluation of the inversion formula (4) with respect to the given data $\left\{d_{j}\right\}$ (e.g. stabilized evaluation of inversion formulas ( $c f$. Anderssen [3])). It can also be applied indirectly by evaluating linear functionals defined on the solution $g(\alpha)$ of the following form

$$
L_{\theta}(g)=\int_{0}^{\pi / 2} \theta(\alpha) g(\alpha) d \alpha, \quad \theta(\alpha) \text { known, }
$$

instead of $g(\alpha)$ itself. (N.B. The function $\theta$ in (6) is not related to the $\theta$ in Miller [11].) This latter idea has been applied to a number of first kind integral equations 
(cf. Golberg [9] and Anderssen [4]). The aim of the present paper is to explore the use of this linear functional approach in the study of contact frequency data.

Often, for inference purposes, the practitioner is more interested in functionals of the form (6) than in $g(\alpha)$ itself ( $c f$. Golberg [9]). Examples of such functionals which arise in the study of foliage angle density are discussed in Section 2.

A number of computational procedures have been proposed for the leaf area index functional

$$
F=\int_{0}^{\pi / 2} g(\alpha) d \alpha
$$

Philip [13] proposed a Fourier series method for determining $F$ based on first smoothing the data to "recover" $f$ then solving the integral equation for $g$, and finally evaluating (7). It is of interest to note that this method can be given a Wiener filtering interpretation and that in terms of this interpretation a formalization for Philip's approach can be obtained ( $c f$. Anderssen and Bloomfield [2]). The disadvantage of this approach is that its implementation requires implicitly that estimates of the first, second and third derivatives of $f(\beta)$ be derived from the smoothed data.

On the basis of the above remarks about the improperly posed nature of (1) and (2), it is essential to seek procedures for evaluating $F$ which involve the lowest possible order of differentiation of the data. In fact, Miller [12] observed that, by exploiting the existence of the inversion formula (4), $F$ can be expressed as a linear functional of $f$ of the form

$$
F=2 \int_{0}^{\pi / 2} \cos \beta f(\beta) d \beta
$$

and consequently can be computed directly from the data $\left\{d_{j}\right\}$ without differentiation.

This result is a special case of a more general result connected with the linear functionals (6). Under appropriate regularity conditions on $\theta(\alpha)$, the linear functionals (6) are equivalent to linear functionals $M(f)$ defined on the contact frequency $f(\beta)$. Through the use of generalized functions, a representation of the form

$$
L_{\varphi}(f)=\int_{0}^{\pi / 2} \varphi(\beta) f(\beta) d \beta
$$

might be used, but this will not be pursued here, except for the Comment of Section 4. The general solution is given by Golberg [9], while the special situation for Abel integral equations along with some general results for integral equations is contained in Anderssen [4]. 
This is the appeal of the linear functional approach. The inherent sensitivity in the definition of $g(\alpha)$ is annihilated or reduced through the construction of a functional defined exclusively on the data $f(\beta)$.

In this paper, we show how this general result can be constructed for the functionals (6). It hinges on the construction in Section 3 of an alternative inversion formula to that given by (4). The basic transform from (6) to $M(f)$ is constructed in Section 4. In Section 5, it is applied to the first three functionals listed in Section 2. Because the fourth functional in Section 2 does not satisfy the conditions of the theorem given in Section 4, it is necessary to construct the transformation for it by different means. This is also done in Section 5.

\section{Linear functionals of foliage angle density}

Some examples of linear functionals which arise in the study of foliage angle density are:

1. Leaf area index or foliage density (i.e. the area of foliage per unit volume of space) (cf. Warren Wilson [23], page 96; Miller [12])

$$
F=\int_{0}^{\pi / 2} g(\alpha) d \alpha, \quad \theta(\alpha) \equiv 1
$$

The term foliage density is used when $g(\alpha)$ is only defined for a specific level in the canopy (such data are obtained from inclined point quadrats), while the term leaf area index is used when either the foliage densities are summed for the whole canopy or $g(\alpha)$ is defined for the whole canopy (such data are obtained when photographic methods are used).

2. Vertical projector of the leaf area index ( $c f$. Suits [19])

$$
L_{\sin \alpha}(g)=\int_{0}^{\pi / 2} \sin \alpha g(\alpha) d \alpha, \quad \theta(\alpha)=\sin \alpha .
$$

3. Horizontal projector of the leaf area index ( $c f$. Suits [19])

$$
L_{\cos \alpha}(g)=\int_{0}^{\pi / 2} \cos \alpha g(\alpha) d \alpha, \quad \theta(\alpha)=\cos \alpha .
$$

4. The segmented foliage density ( $c f$. Ross [15], Smith and Oliver [16])

$$
L_{\chi[a, b]}(g)=\int_{0}^{\pi / 2} \chi[a, b] g(\alpha) d \alpha, \quad 0 \leqslant a \leqslant b \leqslant \pi / 2,
$$


with

$$
\theta(\alpha)=\chi[a, b]= \begin{cases}1, & \alpha \in[a, b] \\ 0, & \alpha \notin[a, b]\end{cases}
$$

In forest research, wide use is made of leaf area index and foliage density. For example, it has been used extensively in the study of the photosynthesis of canopies and the effects of older leaves (cf. de Wit [7]). The vertical and horizontal projectors of the leaf area index play a fundamental role in radiative transfer models of light through a canopy ( $c f$. Suits [19]). The segmented foliage density is useful when the amount of data is limited and only a rough (qualitative) picture of the structure of $g(\alpha)$ is required, such as a three step density model of the type used extensively by Ross [15]. It can also be used to evaluate the leaf distribution function ( $c f$. Smith and Oliver [16])

$$
G(\beta)=L_{x[0, \beta]}(g)=\int_{0}^{\beta} g(\alpha) d \alpha, \quad 0<\beta \leqslant \pi / 2 .
$$

More complex functionals are used in the study of directional reflectance and the penetration of light into canopies ( $c f$. Anderssen, Jackett and Jupp [5]).

\section{An alternative inversion formula}

It is well known that the Abel integral equation

$$
s(x)=\int_{x}^{a} \frac{2 r u(r)}{\sqrt{r^{2}-x^{2}}} d r, \quad 0 \leqslant x \leqslant a \leqslant \infty, \quad a=\text { constant }
$$

has two inversion formulas ( $c f$. Sneddon [18])

$$
u(r)=-\frac{1}{\pi} \int_{r}^{a} \frac{s^{\prime}(x)}{\sqrt{x^{2}-r^{2}}} d x, \quad s^{\prime}(x)=d s(x) / d x,
$$

and

$$
u(r)=-\frac{1}{\pi r} \frac{d}{d r}\left\{\int_{r}^{a} \frac{x s(x)}{\sqrt{x^{2}-r^{2}}} d x\right\}
$$

and that it is via the use of this second inversion formula that the transformation of linear functionals defined on $u(r)$ to linear functionals defined on $s(x)$ is 
accomplished. It is therefore natural to seek the corresponding alternative to the inversion formula (4). It is given by

Lemma. If $f$ is such that $f^{\prime \prime}$ exists and is absolutely continuous on $[0, \pi / 2]$ and $f^{\prime}(0)=f^{\prime}(\pi / 2)=0$, then

$$
g(\alpha)=-\sec \alpha \frac{d}{d \alpha}\left\{\int_{\alpha}^{\pi / 2} \frac{\sin \beta\left(f(\beta)+f^{\prime \prime}(\beta)\right)}{\left(\tan ^{2} \hat{\beta}-\tan ^{2} \alpha\right)^{1 / 2}} d \beta\right\}
$$

exists for almost all $\alpha$ and is the solution of (1) belonging to $L_{1}$.

Proof. Applying integration by parts to the right hand side of (14) yields

$$
\begin{aligned}
\text { r.h.s. (14) } & =-\sec \alpha \frac{d}{d \alpha}\left\{\int_{\alpha}^{\pi / 2} \cos ^{3} \beta\left(f(\beta)+f^{\prime \prime}(\beta)\right) \frac{d}{d \beta}\left(\tan ^{2} \beta-\tan ^{2} \alpha\right)^{1 / 2} d \beta\right\} \\
& =\sec \alpha \frac{d}{d \alpha}\left\{\int_{\alpha}^{\pi / 2}\left(\tan ^{2} \beta-\tan ^{2} \alpha\right)^{1 / 2} \frac{d}{d \beta}\left(\cos ^{3} \beta\left(f(\beta)+f^{\prime \prime}(\beta)\right)\right) d \beta\right\},
\end{aligned}
$$

and hence

$$
\begin{aligned}
\text { r.h.s. (14) }= & -\tan \alpha \sec ^{3} \alpha \\
& \times \int_{0}^{\pi / 2} \frac{d}{d \beta}\left(\cos ^{3} \beta\left(f(\beta)+f^{\prime \prime}(\beta)\right)\right)\left(\tan ^{2} \beta-\tan ^{2} \alpha\right)^{-1 / 2} d \beta
\end{aligned}
$$

The result follows since this last expression is simply a more compact form of the right hand side of (4).

\section{Transformation of linear functionals defined on the foliage angle density}

We now construct the general transformation for the functionals $L_{\theta}(g)$ of (6).

THEOREM 1. Let $f$ be such that $f^{\prime \prime}$ exists and is absolutely continuous on $[0, \pi / 2]$ and $f^{\prime}(0)=f^{\prime}(\pi / 2)=0$, and assume that $\theta$ is a given function on $[0, \pi / 2]$ such that $\theta^{\prime}$ exists and is continuous. Then the linear functionals

$$
L_{\theta}(g)=\int_{0}^{\pi / 2} \theta(\alpha) g(\alpha) d \alpha
$$

can be transformed to

$$
L_{\theta}(g)=\theta(0) f(\pi / 2)+\int_{0}^{\pi / 2} \psi(\beta) \sin \beta\left\{f(\beta)+f^{\prime \prime}(\beta)\right\} d \beta=M(f)
$$


with

$$
\psi(\beta)=\int_{0}^{\beta} \frac{d}{d \alpha}(\sec \alpha \theta(\alpha))\left(\tan ^{2} \beta-\tan ^{2} \alpha\right)^{-1 / 2} d \alpha
$$

PROOF. Substitution of (14) into (15) yields

$$
L_{\theta}(g)=-\int_{0}^{\pi / 2} \sec \alpha \theta(\alpha) \frac{d \tau}{d \alpha} d \alpha
$$

with

$$
\tau(\alpha)=\int_{\alpha}^{\pi / 2} \sin \beta\left(f(\beta)+f^{\prime \prime}(\beta)\right)\left(\tan ^{2} \beta-\tan ^{2} \alpha\right)^{-1 / 2} d \beta
$$

Applying integration by parts to (18), we obtain

$$
\begin{aligned}
L_{\theta}(g) & =-[\sec \alpha \theta(\alpha) \tau(\alpha)]_{\alpha=0}^{\alpha=\pi / 2}+\int_{0}^{\pi / 2} \tau(\alpha) \frac{d}{d \alpha}(\sec \alpha \theta(\alpha)) d \alpha \\
& =\theta(0) \int_{0}^{\pi / 2} \cos \beta\left(f(\beta)+f^{\prime \prime}(\beta)\right) d \beta+\int_{0}^{\pi / 2} \tau(\alpha) \frac{d}{d \alpha}(\sec \alpha \theta(\alpha)) d \alpha .
\end{aligned}
$$

After applying integration by parts twice to the term

$$
\int_{0}^{\pi / 2} \cos \beta f^{\prime \prime}(\beta) d \beta
$$

and using the fact that $f^{\prime}(0)=0$, this last expression becomes

$$
L_{\theta}(g)=\theta(0) f(\pi / 2)+\int_{0}^{\pi / 2} \tau(\alpha) \frac{d}{d \alpha}(\sec \alpha \theta(\alpha)) d \alpha .
$$

The required result (16) now follows on applying a change in the order of integration to this last expression.

As an immediate consequence, it follows that, in terms of the order of differentiation of $f(\beta)$ required, (16) is better posed numerically for the evaluation of $L_{\theta}$ than (15). The order of differentiation which must be applied to $f(\beta)$ can be further reduced if $\psi(\beta)$ is sufficiently smooth. In fact, we have

CoROLLARY 1. If $\psi^{\prime}(\beta)$ exists and is integrable on $[0, \pi / 2]$, then

$$
M(f)=\theta(0) f(\pi / 2)+\int_{0}^{\pi / 2}\left\{\psi(\beta) \sin \beta f(\beta)-f^{\prime}(\beta) \frac{d}{d \beta}(\psi(\beta) \sin \beta)\right\} d \beta \text {. }
$$

If, further, $\psi^{\prime}(\beta)$ is absolutely continuous on $[0, \pi / 2]$, then

$$
\begin{aligned}
M(f)= & \left\{\theta(0)-\psi^{\prime}(\pi / 2)\right\} f(\pi / 2)+\psi(0) f(0) \\
& +\int_{0}^{\pi / 2}\left\{\psi(\beta) \sin \beta+\frac{d^{2}}{d \beta^{2}}(\psi(\beta) \sin \beta)\right\} f(\beta) d \beta .
\end{aligned}
$$


Proof. Apply integration by parts to the term

$$
\int_{0}^{\pi / 2} \psi(\beta) \sin \beta f^{\prime \prime}(\beta) d \beta
$$

on the right hand side of (16).

COMment. There are a number of ways in which the linear functionals (6) can be interpreted within a broader mathematical framework. Whereas the argument used here exploits the existence of explicit inversion formulas, the essence of the argument used by Golberg [9] exploits the inner product representation for bounded linear functionals in the Hilbert space of $L_{2}$-functions defined on $(0, \pi / 2)$; namely

$$
L_{\theta}(g)=\int_{0}^{\pi / 2} \theta(\alpha) g(\alpha) d \alpha=(\theta, g), \quad \theta, g \in L_{2}(0, \pi / 2),
$$

where (.,.) denotes the inner product in $L_{2}(\pi / 2)$.

On the other hand, we know from Miller [11] that the Fredholm integral equation (1) is defined for all $g(\alpha) \in L_{1}(0, \pi / 2)$ (the Banach space of measurably and absolutely integrable (in the Lebesgue sense) functions on $(0, \pi / 2)$ ). This space can therefore be taken as the domain of the operator defined by (1). In this way, the bounded linear functionals $L_{\theta}(g)$ defined on $g$ can be interpreted as bounded linear functionals on $L_{1}$. It is well known that the functions $\theta$ which define these functionals generate the dual space of $L_{1}(0, \pi / 2)$ which is the space $L_{\infty}(0, \pi / 2)$. This yields an alternative characterization for the functions $\theta$ and the functionals they generate.

\section{Transformations for some specific linear functionals of foliage angle density}

We now apply the result of Section 4 to transform the leaf area index and vertical and horizontal projectors of the leaf area index to linear functionals defined on the contact frequency $f(\beta)$.

Initially, we derive the formula for the leaf area index $F$ which was first obtained by Miller [11].

COROLlaRy 2. For $\theta(\alpha)=1$,

$$
\psi(\beta)=\beta
$$

and the leaf area index $F$ of $(7)$ becomes

$$
F=2 \int_{0}^{\pi / 2} \cos \beta f(\beta) d \beta .
$$


Proof. Introducing the change of variable $\tan \beta=s$ and $\tan \alpha=t$ after setting $\theta(\alpha) \equiv 1,(17)$ becomes

$$
\psi(\beta)=\int_{0}^{s} \frac{t d t}{\sqrt{1+t^{2}} \sqrt{s^{2}-t^{2}}} .
$$

A further change of variables defined by $s^{2}=w$ and $t^{2}=v$ yields an integral tabulated in Erdélyi et al. [8], 13.1(8), from which it follows that

$$
\psi(\beta)=w^{1 / 2}{ }_{2} F_{1}(1,1 / 2 ; 3 / 2 ;-w) .
$$

Using appropriate results about hypergeometric functions (cf. Abramowitz and Stegun [1], 15.1.1 and 15.1.5), it follows that

$$
\psi(\beta)=\beta \text {. }
$$

The required result (8) now follows on substituting this value for $\psi(\beta)$ in (20).

COROllary 3. For $\theta(\alpha)=\sin \alpha$,

$$
\psi(\beta)=\pi / 2
$$

and the vertical projector of leaf area index (10) becomes

$$
L_{\sin \alpha}(g)=\pi f(0) / 2 \text {. }
$$

Proof. Introducing the change of variables $\tan \beta=s$ and $\tan \alpha=t$ after setting $\theta(\alpha)=\sin \alpha,(17)$ becomes

$$
\psi(\beta)=\int_{0}^{s} \frac{d t}{\left(s^{2}-t^{2}\right)^{1 / 2}}=\pi / 2 .
$$

The required result (23) now follows on substituting this value for $\psi(\beta)$ in (20).

Corollary 4. For $\theta(\alpha)=\cos \alpha$,

$$
\psi(\beta)=0
$$

and the horizontal projector of leaf area index (11) becomes

$$
L_{\cos \alpha}(g)=f(\pi / 2) \text {. }
$$

For the segmented foliage density (12),

$$
\theta(\alpha)=\chi[a, b], \quad 0 \leqslant a \leqslant b \leqslant \pi / 2,
$$

which does not satisfy the regularity conditions imposed on $\theta(\alpha)$ in Theorem 1 . Nevertheless, the functionals (12) can be transformed. For obvious reasons, it is only necessary to consider the functionals

$$
\theta(\alpha)=\chi[a, \pi / 2], \quad 0 \leqslant a \leqslant \pi / 2 .
$$


By extending the argument Miller [12] used to derive the alternative form (8) for the leaf area index $F$, we obtain

THEOREM 2. The segmented foliage density (12) with $\theta(\alpha)=\chi[a, \pi / 2], 0 \leqslant a \leqslant$ $\pi / 2$, has the following representation as a linear functional (9) defined on the contact frequency $f(\beta)$

$$
L_{x[a, \pi / 2]}(\tilde{b})=\int_{a}^{\pi / 2}(\tau(a, \beta) \sin \beta+\cos \beta \partial \tau(a, \beta) / \partial \beta)\left(f(\beta)+f^{\prime \prime}(\beta)\right) d \beta,
$$

where

$$
\tau(a, \beta)=\arctan \left(\left(\sin ^{2} \beta-\sin ^{2} a\right)^{1 / 2}(\cos \beta)^{-1}\right) .
$$

Proof. Substitution of (4) into (12) yields

$$
\begin{aligned}
L_{x[a, \pi / 2\}}(g)= & -\int_{a}^{\pi / 2} \tan \alpha(\cos \alpha)^{-3} \int_{\alpha}^{\pi / 2}\left(\tan ^{2} \beta-\tan ^{2} \alpha\right)^{-1 / 2} \\
& \times \frac{d}{d \beta}\left\{\cos ^{3} \beta\left(f(\beta)+f^{\prime \prime}(\beta)\right)\right\} d \beta d \alpha .
\end{aligned}
$$

Changing the order of integration, we obtain

$$
L_{x[a, \pi / 2]}(g)=-\int_{a}^{\pi / 2} I(\beta) \frac{d}{d \beta}\left\{\cos ^{3} \beta\left(f(\beta)+f^{\prime \prime}(\beta)\right\} d \beta,\right.
$$

where

$$
\begin{aligned}
I(\beta) & =\int_{a}^{\beta} \tan \alpha(\cos \alpha)^{-3}\left(\tan ^{2} \beta-\tan ^{2} \alpha\right)^{-1 / 2} d \alpha \\
& =\cos \beta \int_{a}^{\beta} \sin \alpha(\cos \alpha)^{-3}\left(\sin ^{2} \beta-\sin ^{2} \alpha\right)^{-1 / 2} d \alpha,
\end{aligned}
$$

because

$$
\tan ^{2} \beta-\tan ^{2} \alpha=\left(\sin ^{2} \beta-\sin ^{2} \alpha\right) / \cos ^{2} \alpha \cos ^{2} \beta .
$$

The introduction of the change of variable

$$
x^{2}=\sin ^{2} \beta-\sin ^{2} \alpha
$$

into (29) yields

$$
I(\beta)=\cos \beta \int_{0}^{z}\left(x^{2}+\cos ^{2} \beta\right)^{-2} d x, \quad z=\left(\sin ^{2} \beta-\sin ^{2} a\right)^{1 / 2} .
$$

This last integral is tabulated in Abramowitz and Stegun [1], equation 3.3.24. Using this result, $I(\beta)$ becomes

$$
I(\beta)=\tau(a, \beta)\left(2 \cos ^{2} \beta\right)^{-1}+\left(\sin ^{2} \beta-\sin ^{2} a\right)^{1 / 2}\left(2 \cos \beta \cos ^{2} a\right)^{-1}
$$

with $\tau(a, \beta)$ defined as in (27). 
We note that, when $a=0,(30)$ yields

$$
I(\beta)=\left(\beta \sec ^{2} \beta+\tan \beta\right) / 2,
$$

which agrees with Miller [12].

We next observe that the application of integration by parts to (28) yields

$$
L_{x[a, \pi / 2]}(g)=\int_{a}^{\pi / 2} I^{\prime}(\beta) \cos ^{3} \beta\left(f(\beta)+f^{\prime \prime}(\beta)\right) d \beta,
$$

since

$$
I(\beta) \cos ^{3} \beta=\frac{1}{2} \cos \beta \tau(a, \beta)+\frac{1}{2} \cos ^{2} \beta\left(\sin ^{2} \beta-\sin ^{2} a\right)^{1 / 2}\left(\cos ^{2} a\right)^{-1}
$$

vanishes when $\beta=a$ and $\beta=\pi / 2$. Standard manipulations yield

$$
\partial \tau(a, \beta) / \partial \beta=\sin \beta\left(\sin ^{2} \beta-\sin ^{2} a\right)^{-1 / 2}
$$

and

$$
I^{\prime}(\beta)=\{\cos \beta \partial \tau(a, \beta) / \partial \beta+\sin \beta \tau(a, \beta)\} / \cos ^{3} \beta .
$$

The required result (26) follows on substituting (32) in (31).

In conclusion, we note that the type of results derived here will also hold for the other foliage angle integral equations examined by Philip [14].

\section{Acknowledgments}

The authors wish to thank David Jupp, CSIRO Division of Water and Land Resources, for drawing their attention to the problem discussed in this paper, and one of the referees who made a number of helpful suggestions and comments.

\section{References}

[1] M. Abramowitz and I. A. Stegun (eds.), Handbook of mathematıcal functions (Dover, New York, 1965).

[2] R. S. Anderssen and P. Bloomfield, "A time series approach to numerical differentiation", Technometrics 16 (1974), 69-75.

[3] R. S. Anderssen, "Stable procedures for the inversion of Abel's equation", J. Inst. Math. Appl. 17 (1976), 329-342.

[4] R. S. Anderssen, "On the use of linear functionals for Abel-type integral equations in applications", in The application and numerical solution of integral equations (eds. R. S. Anderssen, F. R. de Hoog and M. A. Lukas), (Sijthoff and Noordhoff, The Netherlands, 1980).

[5] R. S. Anderssen, D. R. Jackett and D. L. Jupp, "On the use of linear functionals of the foliage angle distribution in the study of the architecture of plant canopies", submitted to Austral. J. Bot. 
[6] F. R. de Hoog, "Review of Fredholm equations of the first kind", in The application and numerical solution of integral equations (eds. R. S. Anderssen, F. R. de Hoog and M. A. Jukas), (Sijthoff and Noordhoff, The Netherlands, 1980).

[7] C. T. de Wit, "Photosynthesis of leaf canopies", Argiculture Research Report No. 663, Centre for Argicultural Publications and Documentation, Wageningen, The Netherlands (1965).

[8] A. Erdélyi et al., Tables of integral transforms-Bateman manuscript project (Vol. 2, McGraw-Hill, New York, 1954).

[9] M. A. Golberg, “A method of adjoints for solving some ill-posed equations of the first kind”, Appl. Math. Comp. 5 (1979). 123-130.

[10] M. A. Lukas, "Regularization" in The application and numerical solution of integral equations (eds. R. S. Anderssen, F. R. de Hoog and M. A. Lukas), (Sijthoff and Noordhoff, The Netherlands, 1980).

[11] J. B. Miller, “An integral equation from phytology”, J. Austral. Math. Soc. 4 (1963), 397-402.

[12] J. B. Miller, “A formula for average foliage density", Austral. J. Bot 15 (1967), 141-144.

[13] J. R. Philip, "The distribution of foliage density with foliage angle estimated from inclined point quadrat observations", A ustral. J. Bot. 13 (1965), 357-366.

[14] J. R. Philip, "Some integral equations in geometrical probability", Btometrık 53 (1966), 365-374.

[15] J. Ross, The radiation regime and the architecture of plant stands (W. Junk, The Hague, 1981).

[16] J. A. Smith and R. E. Oliver, "Effects of changing canopy directional reflectance on feature selection", Appl. Optics 13 (1974), 1599-1604.

[17] J. A. Smith, R. E. Oliver and J. K. Berry, "A comparison of two photographic techniques for estimating foliage angle distribution", Austral. J. Bot. 25 (1977), 545-553.

[18] I. N. Sneddon, Mixed boundary value problems in potential theory (North-Holland, Amsterdam, 1966).

[19] G. H. Suits, "The calculation of the directional reflectance of a vegetative canopy", Rem. Sens. Environ. 2(1972), 117-125.

[20] G. Wahba, "Practical approximate solutions to linear operator equations when the data are noisy", SIAM J. Numer. Anal. 14 (1977), 651-667.

[21] G. Wahba, "Ill-posed problems: numerical and statistical method for mildly, moderately and severely ill-posed problems with noisy data", University of Wisconsin-Madison, Department of Statistics, Technical Report \#595 (1980).

[22] J. Warren Wilson, "Inclined point quadrats" (with Appendix by J. E. Reeve), The New Phytologist 59 (1960), 1-8.

[23] J. Warren Wilson, "Estimation of foliage denseness and foliage angle by inclined point quadrats", A ustral. J. Bot. 11 (1963), 95-105. 